\title{
Analysis on the Luminous Efficiency of Phosphor-Conversion White Light-Emitting Diode
}

\author{
Han-Youl Ryu* \\ Department of Physics, Inha University, Incheon 402-751, Korea
}

(Received October 30, 2012 : revised December 28, 2012 : accepted January 10, 2013)

\begin{abstract}
The author analyzes the luminous efficiency of the phosphor-conversion white light-emitting diode (LED) that consists of a blue LED chip and a yellow phosphor. A theoretical model is derived to find the relation between luminous efficiency (LE) of a white LED, wall-plug efficiency (WPE) of a blue LED chip, and the phosphor absorption ratio of blue light. The presented model enables to obtain the theoretical limit of LE and the lower bound of WPE. When the efficiency model is applied to the measured results of a phosphor-conversion white LED, the limit theoretical value of LE is obtained to be $261 \mathrm{~lm} / \mathrm{W}$. In addition, for LE of $88 \mathrm{~lm} / \mathrm{W}$ at $350 \mathrm{~mA}$, the lower bound of WPE in the blue LED chip is found to be $\sim 34 \%$. The phosphor absorption ratio of blue light was found to have an important role in optimizing the luminous efficiency and colorimetric properties of phosphor-conversion white LEDs.
\end{abstract}

Keywords: Light-emitting diode, White LED, Phosphor, Luminous efficiency

OCIS codes : (230.3670) Light-emitting diodes; (260.3800) Luminescence; (330.1710) Color measurement

\section{INTRODUCTION}

Recently, solid-state lighting based on white light-emitting diodes (LEDs) has attracted great interest as a high-energyefficiency and environment-friendly lighting technology [1-4]. Now, the luminous efficiency (LE) of white LEDs far exceeds that of incandescent and fluorescent lamps, which enables the white LEDs to be used in various lighting applications such as back lights in liquid-crystal displays, automobile lamps, and general illumination. By further increasing the luminous efficiency and lowering the production cost, the white LEDs are expected to widely replace current light bulbs in the near future.

Currently, most white LEDs are manufactured as a phosphorconversion type, which consists of a $\mathrm{GaN} / \mathrm{InGaN}$-based blue LED and a yellow phosphor excited by the blue LED. In this phosphor-conversion white LED, part of blue light emitted from the blue LED chip is absorbed in the phosphor and converted into yellow light from the phosphor. The unabsorbed blue light is transmitted out of the phosphor. By properly mixing the yellow light of the phosphor and the transmitted blue light, white light with moderate chromaticity coordinates and correlated color temperature (CCT) can be produced. During the last several years, there has been rapid progress in the luminous efficiency of the phosphorconversion white LEDs. The luminous efficiency (LE) of commercially available white LEDs can be higher than $120 \mathrm{~lm} / \mathrm{W}$, and that of R\&D LED samples has been reported to be as high as $180 \mathrm{~lm} / \mathrm{W}$ [5].

However, there has been lack of study on the theoretical analysis of LE in the phosphor-conversion white LEDs. The analysis of LE in the phosphor-conversion white LED is not so simple compared with that in the multi-chip-based white LEDs owing to the conversion processes in phosphors. Although the theoretical limit of LE in phosphor-conversion white LEDs has been believed to be $260 \sim 300 \mathrm{~lm} / \mathrm{W}$ [5], theoretical models to obtain the limit theoretical value of LE have not been clearly presented. In this paper, we rigorously analyze LE of phosphor-conversion white LEDs based on the photometric study of white light. The analytical model is used to find the theoretical limit of LE using the measured spectrum of the phosphor-conversion white LED. It will be shown that the presented model can be used to obtain some information on the efficiency of the measured white LED such as the radiative conversion efficiency of the phosphor and the wall-plug efficiency (WPE) of the blue LED chip used in the phosphor-conversion white LED.

\footnotetext{
*Corresponding author: hanryu@inha.ac.kr

Color versions of one or more of the figures in this paper are available online.
} 


\section{THEORY AND ANALYSIS}

LE of an LED, $\eta_{l m}$ is defined as the luminous flux of the LED divided by the electrical input power.

$$
\eta_{l m} \equiv \frac{\Phi_{l m}}{I V}
$$

where $I$ and $V$ are current and voltage applied to the LED, and $\Phi_{l m}$ is the luminous flux. LE can also be written as below using WPE, $\eta_{\text {WPE }}$ and output power of the LED chip, $P_{\text {chip }}$.

$$
\eta_{l m}=\eta_{W P E} \frac{\Phi_{l m}}{P_{c h i p}}
$$

For phosphor-conversion white LEDs, the luminous flux is express as

$$
\Phi_{l m}=683[\mathrm{~lm} / \mathrm{W}] \times \int V(\lambda)\left[P_{b}(\lambda)+P_{y}(\lambda)\right] d \lambda
$$

where $I$ is the wavelength light and $V(\lambda)$ is the eye sensitivity function. $V(\lambda)$ is a dimensionless quantity which is normalized to 1 at $555 \mathrm{~nm} . P_{b}(\lambda)$ and $P_{y}(\lambda)$ are the power spectral density of the blue and the yellow light, respectively, which can be obtained by the spectral measurement of the white LED. Then, output power of the blue light, $P_{b}$ and the yellow light, $P_{y}$ from the white LED is respectively given by

$$
P_{b}=\int P_{b}(\lambda) d \lambda \text { and } P_{y}=\int P_{y}(\lambda) d \lambda
$$

We denote $\alpha$ as the ratio of the absorbed power at the phosphor to the output power from the blue LED chip. Then, the absorbed power of blue light at the phosphor, $P_{a b s}$ and the transmitted output power of blue light, $P_{b}$ are given by

$$
P_{a b s}=\alpha P_{c h i p} \text { and } P_{b}=(1-\alpha) P_{c h i p}
$$

Consequently, LE is written as

$$
\eta_{l m}=683[1 \mathrm{~m} / \mathrm{W}] \times \eta_{W P E} \frac{(1-\alpha) \int V(\lambda)\left[P_{b}(\lambda)+P_{y}(\lambda)\right] d \lambda}{\int P_{b}(\lambda) d \lambda}
$$

Eq. (6) relates LE of the phosphor-conversion white LED with WPE of the blue LED chip inside the white LED. Since $\eta_{l m}$ can be experimentally measured, $\eta_{W P E}$ of the LED chip can be determined if $\alpha$ is known.
Here, we introduce a quantity, $R$ which is defined as the ratio of the yellow light power to the blue light power.

$$
R \equiv \frac{P_{y}}{P_{b}}
$$

By using Eq. (5), $R$ can be expressed as

$$
R=\frac{\alpha}{1-\alpha} \frac{P_{y}}{P_{a b s}}
$$

$P_{a b s}$ can be written as

$$
P_{a b s}=\int P_{y}(\lambda) / \eta_{p h o s}(\lambda) d \lambda
$$

where $\eta_{\text {phos }}(\lambda)$ is the efficiency of the phosphor which includes the conversion efficiency from blue to yellow light (Stokes shift efficiency) and radiative efficiency of the phosphor. Therefore, Eq. (8) is rewritten as

$$
R=\frac{\alpha}{1-\alpha} \frac{\int P_{y}(\lambda) d \lambda}{\int P_{y}(\lambda) / \eta_{p h o s}(\lambda) d \lambda}
$$

$\eta_{\text {phos }}(\lambda)$ can be regarded as the product of the radiative efficiency of a phosphor and the Stokes shift efficiency. That is,

$$
\eta_{\text {phos }}(\lambda)=\eta_{\text {phos }}^{\text {rad }} \eta_{\text {phos }}^{\text {Stoke }}=\eta_{\text {phos }}^{\text {rad }} \frac{\bar{\lambda}_{\text {blue }}}{\lambda}
$$

where $\bar{\eta}_{p h o s}^{\text {rad }}$ is the radiative efficiency of a phosphor which is assumed to be independent of emission wavelength and $\bar{\lambda}_{\text {blue }}$ is the average wavelength of the blue light spectrum. Then, $R$ is given by

$$
R=\left(\frac{\alpha}{1-\alpha}\right) \frac{\eta_{\text {phos }}^{\text {rad }} \bar{\lambda}_{\text {blue }} \int P_{y}(\lambda) d \lambda}{\int \lambda P_{y}(\lambda) d \lambda}
$$

Eq. (12) implies that $\bar{\eta}_{\text {phos }}^{\text {rad }}$ can be determined once $\alpha$ is known since $R, P_{y}(\lambda)$, and $\bar{\lambda}_{\text {blue }}$ are obtained experimentally. However, in order to obtain $\alpha$ from Eq. (6), $\eta_{l m}$ of the white LED and $\eta_{\text {WPE }}$ of the blue LED should be determined simultaneously, which is not an easy task. Instead, when $\bar{\eta}_{\text {phos }}^{\text {rad }}$ is assumed to be $100 \%$, the lowest limit of $\alpha$ can be obtained from Eq. (12). Then, the theoretical limit of $\eta_{l m}$ is determined from Eq. (6) by using the obtained lowest limit value of $\alpha$ and $\eta_{\text {WPE }}$ value of $100 \%$. In this 
way, the limit theoretical value of LE can be obtained once the spectrum of a white LED is given. In addition, when $\eta_{l m}$ of the white LED is measured, the lower bound of $\eta_{\text {WPE }}$ of the blue LED chip can be obtained from Eq. (6).

The theoretical analysis of the LE in phosphor-conversion white LEDs presented in this section will be applied to the experimentally measured results of a specific white LED in the following sections.

\section{EXPERIMENT}

Characteristics of a commercial phosphor-conversion white LED sample were measured by using an LED characterization system with a calibrated integrating sphere. A packaged LED white sample was soldered on a metal printed circuit board (PCB) which was mounted on a heat sink. The temperature of the metal $\mathrm{PCB}$ was maintained at $25{ }^{\circ} \mathrm{C}$ by a temperature control system with a thermo-electric cooler. Then, electro-optical characteristics were measured by applying current and voltage to the LED.

Photometric properties of the white LED were measured under continuous-wave operation up to $350 \mathrm{~mA}$. Fig. 1 shows the measured spectrum of the white LED when injection current is $350 \mathrm{~mA}$. Typical spectral shape of the phosphor-

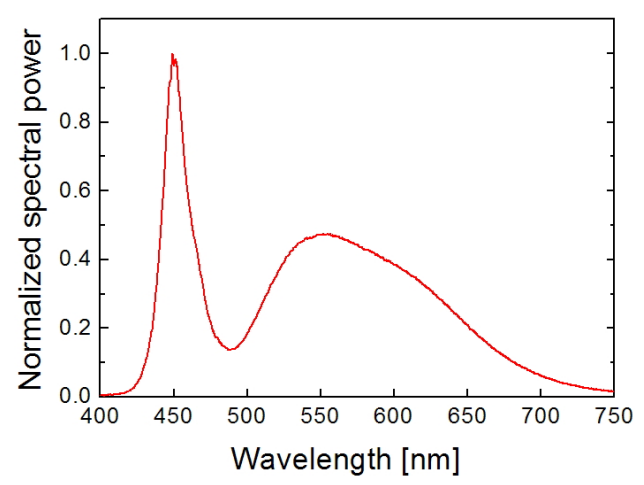

FIG. 1. Spectrum of a commercial phosphor-conversion white LED measured at $25^{\circ} \mathrm{C}$.

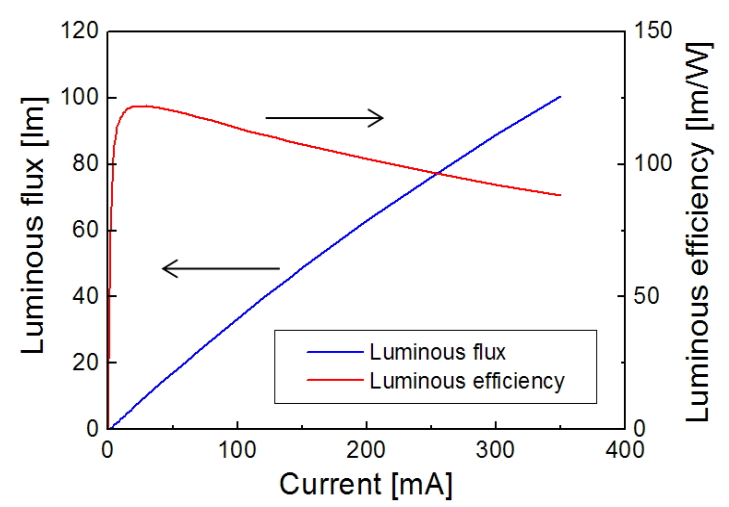

FIG. 2. Luminous flux and luminous efficiency of the white LED as injection current increases up to $350 \mathrm{~mA}$. conversion white LED with a blue LED and a yellow phosphor is observed. In this sample, the peak wavelength of blue light from the InGaN LED chip and yellow light from the phosphor exist around 450 and $555 \mathrm{~nm}$, respectively.

Figure 2 shows the luminous flux and LE of the LED as current increases. The luminous flux increases linearly with injection current and it reaches $\sim 100 \mathrm{~lm}$ at $350 \mathrm{~mA}$. The peak value of LE is $\sim 122 \mathrm{~lm} / \mathrm{W}$ at $30 \mathrm{~mA}$. However, LE decreases gradually with increasing current when current is larger than $30 \mathrm{~mA}$, and it is reduced to $\sim 88 \mathrm{~lm}$ at 350 $\mathrm{mA}$. This decrease in LE of the white LED results mainly from the "efficiency droop" phenomena of the blue LED chip [6-8].

Colorimetric characteristics of the measured white LED were also evaluated based on the standard by Commission International de I'Eclairage (CIE). The chromaticity coordinates in the CIE $x y$ chromaticity diagram were measured to be $(0.33,0.35)$ at $350 \mathrm{~mA}$. And, the CCT and the general color rendering index of the LED were obtained to be $5400 \mathrm{~K}$ and 0.75 , respectively.

\section{RESULTS AND DISCUSSION}

The theoretical limit of LE of the measured phosphorconversion white LED is calculated from Eqs. (6) and (12) using the spectrum in Fig. 1. Assuming that the radiative efficiency of the phosphor, $\bar{\eta}_{p h o s}^{\text {rad }}$ is $100 \%$, the lowest limit of $\alpha$ can be obtained from Eq. (12). $P_{b}$ and $P_{y}$ in Eq. (4) are calculated by integrating the spectral region of the blue and the yellow light, respectively. Then, the ratio of the yellow light power to the blue light power, $R$ is found to be 2.51 from Eq. (7). Using the obtained values of $R$ and $\bar{\lambda}_{\text {blue }}$, the ratio of phosphor absorption, $\alpha$ is calculated to be 0.762 from Eq. (12). Consequently, from Eq. (6), LE is obtained as

$$
\eta_{l m} \leq 261[\mathrm{~lm} / \mathrm{W}] \times \eta_{W P E}
$$

Therefore, the maximum LE of the measured white LED will be $261 \mathrm{~lm} / \mathrm{W}$ assuming that both WPE of the blue LED chip and the radiative efficiency of the yellow phosphor are $100 \%$. By using the measured LE of the white LED in Fig. 2, the lower limit of WPE of the blue LED chip can be deduced from Eq. (13). The peak value of LE at $30 \mathrm{~mA}$ was $122 \mathrm{~lm} / \mathrm{W}$, which corresponds to WPE of $>46.8 \%$. That is, the maximum WPE of the blue LED chip inside the measured white LED is higher than $46.8 \%$. The LE at $350 \mathrm{~mA}$ was measured to be $88 \mathrm{~lm} / \mathrm{W}$, which corresponds to WPE of $>33.7 \%$. Accurate values of WPE can be obtained when the radiative efficiency of the phosphor, $\bar{\eta}_{\text {phos }}^{\text {rad }}$ is known. Conversely, if the WPE of the blue LED chip is known, the radiative efficiency of the phosphor could be determined. 
Eq. (13) implies that the theoretical limit of LE in the measured phosphor-conversion white LED is $261 \mathrm{~lm} / \mathrm{W}$. The theoretical maximum LE can be increased further by optimizing the spectrum of the white LED because LE is strongly influenced by the spectral distribution of emitted light. Eq. (3) implies that good overlap of the yellow light spectrum with the eye sensitivity function is important for increasing LE. In addition, relative output power of the blue and the yellow light also plays an important role in LE. In order to see the effect of the relative ratio of the blue and the yellow light, LE is calculated as a function

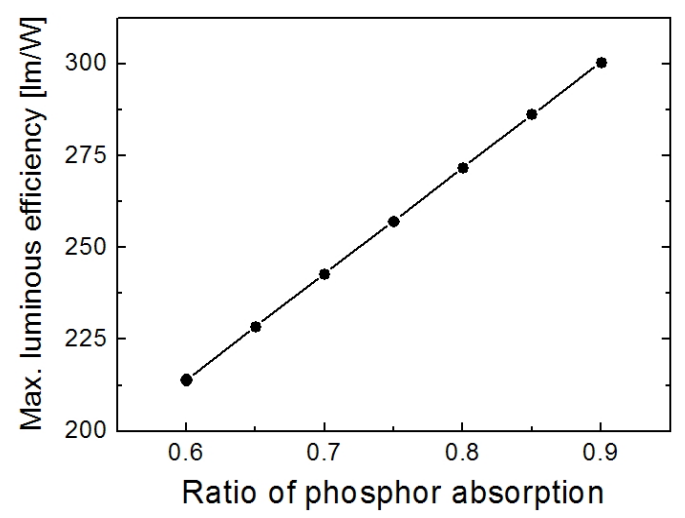

FIG. 3. Theoretical limit of the luminous efficiency in phosphor-conversion white LEDs as a function of the ratio of phosphor absorption, $\alpha$.

(a)

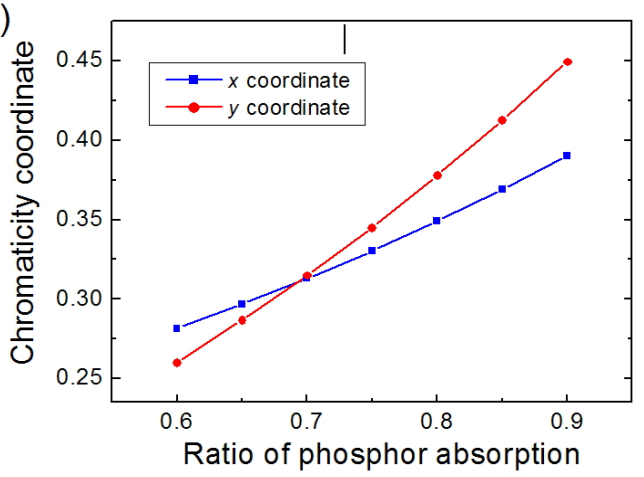

(b)

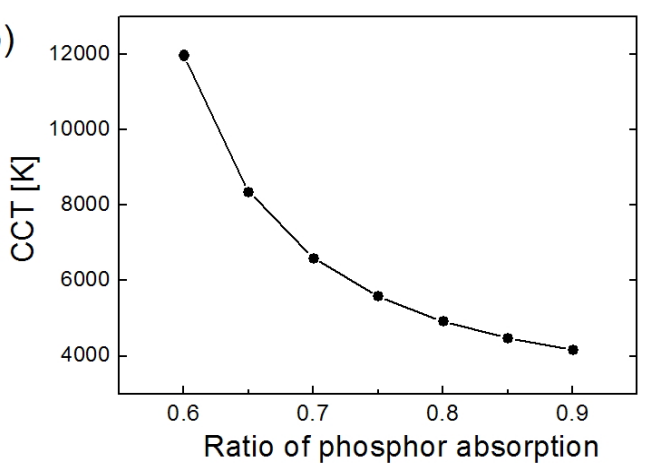

FIG. 4. (a) Chromaticity coordinates in the CIE $x y$ diagram and (b) correlated color temperature in phosphor-conversion white LEDs as a function of the ratio of phosphor absorption, $\alpha$. of the ratio of phosphor absorption, $\alpha$. In this modeling, it is assumed that the blue LED chip and the phosphor are the same as those used in the white LED on which we experimented. So, the spectral distribution of the blue and the yellow light are not changed, and only the relative strength of peak intensity in the blue and the yellow light changes as $\alpha$ varies.

Figure 3 shows the theoretical limit of LE as a function of $\alpha$. The maximum theoretical LE increases linearly from 214 to $300.4 \mathrm{~lm} / \mathrm{W}$ as $\alpha$ increases from 0.6 to 0.9 . Since the yellow light emitted from the phosphor shows much better overlap with the eye sensitivity function than the blue light, increasing the yellow light spectral intensity relative to that of blue light one is advantageous for increasing LE of the white LED. Therefore, higher LE is expected by increasing the phosphor absorption of the blue LED light.

However, $\alpha$ also changes the colorimetric properties of the white LED. For given a LED spectra, chromaticity coordinates and CCT are calculated based on the CIE colorimetric standard [9-12]. Fig. 4 shows the chromaticity coordinates and the CCT as a function of $\alpha$. As $\alpha$ increases, both $x$ and $y$ coordinates in the CIE $x y$ chromaticity diagram increase and the CCT decreases. This is because the chromaticity coordinates shift toward the yellowish region of the chromaticity diagram as the yellow light spectral intensity increases relative to that of the blue light. As shown in Fig. 3, the theoretical limit of LE can be higher than $300 \mathrm{~lm} / \mathrm{W}$ when $\alpha$ is 0.9 . In this case, however, the chromaticity coordinates deviate from the Planckian locus too much, which may not be suitable for general lighting applications. Therefore, it is important to carefully optimize $\alpha$ considering both LE and color quality of the white light. In addition, by further optimizing the spectral distribution of the blue and the yellow light, even higher LE can be achieved with moderate color properties.

\section{SUMMARY}

The LE of the phosphor-conversion white LED was analyzed based on the photometric study of white light. The investigated white LED consists of a GaN-based blue LED chip and a yellow phosphor. We developed a theoretical model describing the relation between LE of a white LED, WPE of a blue LED chip, and the ratio of phosphor absorption of blue light. When the model was applied to a commercial phosphor-conversion white LED, the theoretical limit of LE was obtained to be $261 \mathrm{~lm} / \mathrm{W}$ and the lower bound of WPE in the blue LED chip was found to be $\sim 34 \%$ for $\mathrm{LE}$ of $88 \mathrm{~lm} / \mathrm{W}$ at $350 \mathrm{~mA}$. The theoretical model developed in this work is expected to be used advantageously for optimizing LE and color properties of phosphor-conversion white LEDs. 


\section{ACKNOWLEDGMENT}

This work was supported by the MKE(The Ministry of Knowledge Economy), Korea, under the ITRC(Information Technology Research Center) support program supervised by the NIPA(National IT Industry Promotion Agency)" (NIPA-2012-C1090-1200-0007) and by National Research Foundation of Korea Grant funded by the Korean Government (2012R1A1A2039630).

\section{REFERENCES}

1. E. F. Schubert and J. K. Kim, "Solid-state light sources getting smart," Science 308, 1274-1278 (2005).

2. G. Harbers, S. J. Bierhuizen, and M. R. Krames, "Performance of high power light emitting diodes in display illumination applications," J. Display Technol. 3, 98-108 (2007).

3. G. Chen, M. Craven, A. Kim, A. Munkholm, S. Watanabe, M. Camras, W. Gotz, and F. Steranka, "Performance of high-power III-nitride light emitting diodes," Phys. Status Solidi A 205, 1086-1092 (2008).

4. A. Laubsch, M. Sabathil, J. Baur, M. Peter, and B. Hahn, "High-power and high-efficiency InGaN-based light emitters," IEEE Trans. Electron. Devices 57, 79-87 (2010).
5. Y. Narukawa, M. Ichikawa, D. Sanga, M. Sano, and T. Mukai, "White light emitting diodes with super-high luminous efficacy," J. Phys. D: Appl. Phys. 43, 354002 (2010).

6. J. Piprek, "Efficiency droop in nitride-based light-emitting diodes," Phys. Status Solidi A 207, 2217-2225 (2010).

7. M. H. Crawford, "LEDs for solid-state lighting: performance challenges and recent advances," IEEE J. Select. Topics Quantum Electron. 15, 1028-1040 (2009).

8. H. Y. Ryu, D. S. Shin, and J. I. Shim, "Analysis of efficiency droop in nitride light-emitting diodes by the reduced effective volume of InGaN active material," Appl. Phys. Lett. 100, 131109 (2012).

9. N. Ohta and A. Robertson, Colorimetry: Fundamentals and Applications (Wiley, 2006), Chapter 1 and 3.

10. E. F. Schubert, Light-emitting Diodes (Cambridge University Press, UK, 2006), Chapter 16-18.

11. D. K. Son, E. B. Cho, I. Moon, Y. Park, and C. G. Lee, "Development of an illumination measurement device for color distribution based on a CIE 1931 XYZ sensor," J. Opt. Soc. Korea 15, 44-51 (2011).

12. C. G. Son, J. H. Yi, J. S. Gwag, and J. H. Kwon, and G. Park, "Improvement of color and luminance uniformity of the edge-lit backlight using the RGB LEDs," J. Opt. Soc. Korea 15, 272-277 (2011). 